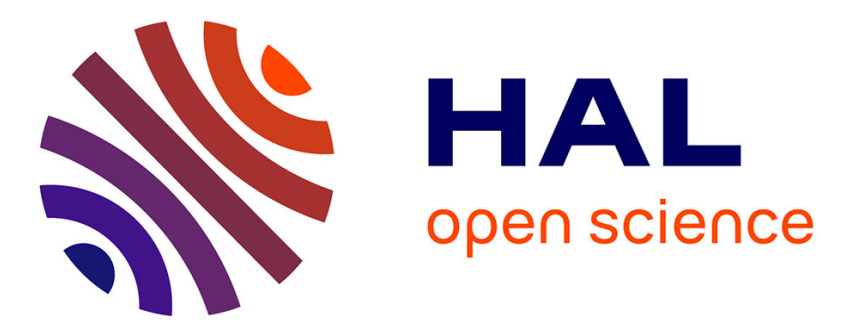

\title{
Analytical and numerical stability analysis of Soret-driven convection in a horizontal porous layer: the effect of conducting bounding plates
}

Bafétigué Ouattara, Ali Khouzam, Abdelkader Mojtabi, Marie-Catherine

Charrier-Mojtabi

\section{To cite this version:}

Bafétigué Ouattara, Ali Khouzam, Abdelkader Mojtabi, Marie-Catherine Charrier-Mojtabi. Analytical and numerical stability analysis of Soret-driven convection in a horizontal porous layer: the effect of conducting bounding plates. Fluid Dynamics Research, 2012, vol. 44, pp. 1-14. 10.1088/01695983/44/3/031415. hal-00866157

\section{HAL Id: hal-00866157 \\ https://hal.science/hal-00866157}

Submitted on 26 Sep 2013

HAL is a multi-disciplinary open access archive for the deposit and dissemination of scientific research documents, whether they are published or not. The documents may come from teaching and research institutions in France or abroad, or from public or private research centers.
L'archive ouverte pluridisciplinaire HAL, est destinée au dépôt et à la diffusion de documents scientifiques de niveau recherche, publiés ou non, émanant des établissements d'enseignement et de recherche français ou étrangers, des laboratoires publics ou privés. 


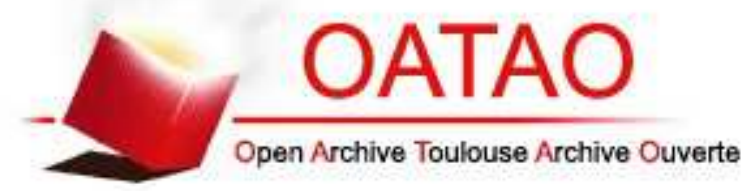

\section{Open Archive TOULOUSE Archive Ouverte (OATAO)}

OATAO is an open access repository that collects the work of Toulouse researchers and makes it freely available over the web where possible.

This is an author-deposited version published in : http://oatao.univ-toulouse.fr/ Eprints ID : 9559

To link to this article : DOI:10.1088/0169-5983/44/3/031415

URL : http://dx.doi.org/10.1088/0169-5983/44/3/031415

To cite this version : Ouattara, Bafétigué and Khouzam, Ali and Mojtabi, Abdelkader and Charrier-Mojtabi, Marie-Catherine. Analytical and numerical stability analysis of Soret-driven convection in a horizontal porous layer: the effect of conducting bounding plates. (2012). Fluid Dynamics Research, vol. 44 $\left(\mathrm{n}^{\circ} 3\right)$. ISSN 0169-5983

Any correspondance concerning this service should be sent to the repository administrator: staff-oatao@ listes-diff.inp-toulouse.fr 


\title{
Analytical and numerical stability analysis of Soret- driven convection in a horizontal porous layer: the effect of conducting bounding plates
}

\author{
B Ouattara ${ }^{1,2}$, A Khouzam ${ }^{1,2}$, A Mojtabi ${ }^{1,2}$ and M C Charrier-Mojtabi ${ }^{3}$ \\ ${ }^{1}$ Université de Toulouse; INPT, UPS; IMFT (Institut de Mécanique des Fluides de Toulouse), \\ Allée Camille Soula, F-31400 Toulouse, France \\ ${ }^{2}$ CNRS, IMFT, F-31400 Toulouse, France \\ ${ }^{3}$ PHASE, EA 810, UFR PCA, Université Paul Sabatier, 118 route de Narbonne, 31062 Toulouse \\ cedex, France
}

E-mail: bouattar@imft.fr, akhouzam@imft.fr,mojtabi@imft.fr and cmojtabi@cict.fr

\begin{abstract}
The aim of this study was to investigate the effect of conducting boundaries on the onset of convection in a binary fluid-saturated porous layer. The isotropic saturated porous layer is bounded by two impermeable but thermally conducting plates, subjected to a constant heat flux. These plates have identical conductivity. Moreover, the conductivity of the plates is generally different from the porous layer conductivity. The overall layer is of large extent in both horizontal directions. The problem is governed by seven dimensionless parameters, namely the normalized porosity of the medium $\varepsilon$, the ratio of plates over the porous layer thickness $\delta$ and their relative thermal conductivities ratio $d$, the separation ratio $\delta$, the Lewis number $L e$ and thermal Rayleigh number $R a$. In this work, an analytical and numerical stability analysis is performed. The equilibrium solution is found to lose its stability via a stationary bifurcation or a Hopf bifurcation depending on the values of the dimensionless parameters. For the long-wavelength mode, the critical Rayleigh number is obtained as $R a_{\mathrm{cs}}=12(1+2 d \delta) /[1+\psi(2 d \delta L e+L e+1)]$ and $k_{\mathrm{cs}}=0$ for $\psi>\psi_{\text {uni }}>0$. This work extends an earlier paper by Mojtabi and Rees (2011 Int. J. Heat Mass Transfer 54 293-301) who considered a configuration where the porous layer is saturated by a pure fluid.
\end{abstract}

(Some figures may appear in colour only in the online journal) 


\section{Introduction}

Double-diffusive convection in a saturated porous medium due to temperature and concentration gradients has been widely studied because of its numerous fundamental and industrial applications. Reviews of recent developments and publications in this field can be found in the books of Nield and Bejan (1999), Ingham and Pop (2005) and Vafaï (2005).

The linear stability of a fluid mixture in a porous medium, in the presence of a temperature gradient and taking into account the Soret effect, was investigated by Ouarzazi and Bois (1994). The temperature gradient was assumed to vary periodically with respect to time. The two-dimensional (2D) instability thresholds, for both oscillatory and stationary instabilities, were predicted by these authors. The onset of Soret-driven convection in an infinite horizontal porous layer heated isothermally from below or from above has been considered by Sovran et al (2001) using a linear stability analysis and nonlinear perturbation theories. A linear stability analysis was used to predict the onset of motion in terms of the buoyancy ratio, the Lewis number and the normalized porosity. Depending on the value of the separation ratio, it was found analytically and numerically that the motionless solution lost its stability via stationary or Hopf bifurcations.

Knobloch and Moore (1988) and Platten et al (2003) studied the onset and development of convection in a horizontal rectangular cavity. The two vertical walls of the cavity are impermeable and adiabatic, while the impermeable horizontal walls are maintained at constant and different temperatures. The authors obtained the stability diagram in which the critical Rayleigh number is given as a function of the separation ratio $\psi$, for a given Lewis number Le. Bahloul et al (2003) studied the stability of the onset of Soret-driven convection. They considered the case when a uniform heat flux is applied to the horizontal impermeable or permeable walls, while the vertical walls are impermeable and adiabatic. The thresholds for finite-amplitude, oscillatory and monotonic convection instabilities were determined in terms of the governing parameters of the problem. This work was extended by Bourich et al (2004) considering not only a shallow horizontal porous cavity but also a shallow enclosure with a binary fluid. The critical Rayleigh numbers for the onset of oscillatory and stationary convections were determined explicitly as functions of the governing parameters for infinite layers and bounded boxes. At the onset of instability, the authors found that the wave number was equal to zero and also showed the dependence of critical parameters on the normalized porosity. Three-dimensional numerical modeling of Soret-driven convection in a cubic cell filled with a binary mixture of water (90\%) and isopropanol (10\%) was performed by Shevtsova et al (2006). Batiste et al (2006) remark that the convections identified in binary mixture convection are located below the onset of absolute instability and hence in a parameter regime, where the instability of the conduction state is only convective. Alonso et al (2007) have presented the results of direct numerical simulations of convection in binary fluids in large-aspect containers. They focused on binary mixtures with negative values of the separation ratio, for which the primary bifurcation is subcritical and oscillatory, and with weak Soret coupling so that nonlinear dispersion is important. Localized stationary convective structures were investigated by Jung and Lücke (2007) through direct numerical simulations in binary fluid mixtures with weakly negative separation ratios. Sparrow et al (1964) and Hurle et al (1967) carried out linear stability analysis of the convection problem with conducting boundaries in pure fluids. Proctor (1981) and Jenkins and Proctor (1984) considered 3D finite-amplitude thermal convection in a fluid layer with boundaries of finite conductivity. They determined the conductivity of the boundaries for which the preferred planform changes from roll to square-cell type. A very few papers exist that consider the effect of the presence of horizontal bounding plates on the onset of thermal convection in saturated porous media (Riahi 1983 and Rees and Mojtabi 2011). 


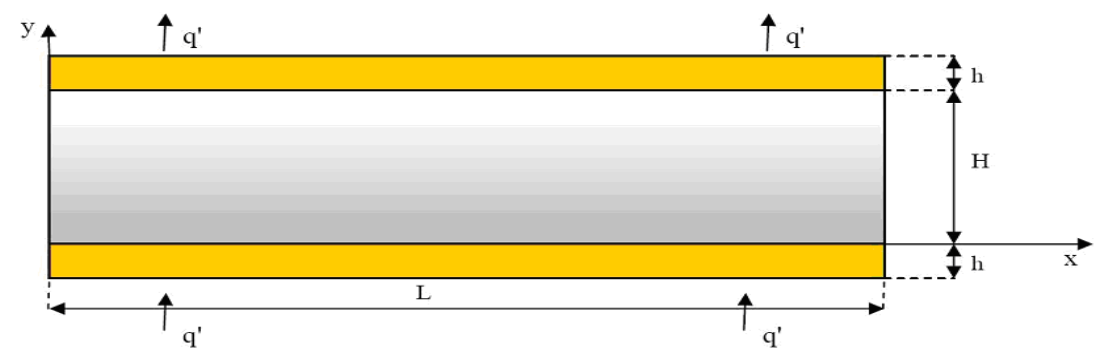

Figure 1. Saturated porous medium of height $H$ and length $L$ bounded by two horizontal plates of thickness $h$. The upper and lower surfaces of the system are subjected to a uniform heat flux. The vertical side walls are assumed to be perfectly insulated.

The main aim of this work, then, is to determine the effect of conducting bounding plates on the stability properties of a horizontal porous layer saturated with a binary fluid. As such, this provides a better approximation of how experiments are set up in the laboratory than do the usual fixed temperature or heat flux boundary conditions. In this paper, particular attention is paid to the influence of the conductivity ratio and thickness ratio.

\section{Mathematical formulation}

The configuration considered in this study is that of a horizontal porous layer of uniform thickness $H$, width $L$, permeability $K$ and porosity $\varepsilon^{*}$, which is filled with a binary fluid mixture (see figure 1). The origin of the coordinate system is located at the bottom of the porous cavity with $x^{\prime}$ and $y^{\prime}$ being the horizontal and vertical coordinates, respectively. This cavity is placed between two metal plates of uniform thickness, $h$. Neumann boundary conditions for temperature (i.e. fixed heat flux) are applied to the outer horizontal surfaces of the layer at $y^{\prime}=-h$ and at $y^{\prime}=H+h$. All the boundaries are rigid and impermeable and we consider a rectangular cavity with a high aspect ratio $A=L / H$.

The impermeable horizontal walls are subjected to a uniform heat flux per unit area, $q^{\prime}$. The vertical walls $\left(x^{\prime}=0, x^{\prime}=L\right)$ are impermeable and adiabatic. We also assume that the porous medium is isotropic and homogeneous, that Darcy's law is valid and that the Oberbeck-Boussinesq approximation is applicable: the thermophysical properties of the binary fluid are therefore considered to be constant except for the density in the buoyancy term, which is taken to vary linearly with the local temperature $T$ and mass fraction $C$ of the denser component,

$$
\rho=\rho_{0}\left[1-\beta_{\mathrm{T}}\left(T^{\prime}-T_{0}\right)-\beta_{\mathrm{C}}\left(C^{\prime}-C_{0}\right)\right],
$$

where $\beta_{\mathrm{T}}$ and $\beta_{\mathrm{C}}$ are, respectively, the thermal and mass expansion coefficients of the binary fluid ( $T_{0}$ and $C_{0}$ correspond to the reference state), $\rho_{0}$ is the fluid mixture reference density at $T_{0}$ and $C_{0}$, and $T^{\prime}$ is the dimensional temperature. We also use other standard assumptions such as local thermal equilibrium between the phases and negligible viscous dissipation.

Thus the governing conservation equations for mass, momentum, energy and chemical species for the bulk are

$$
\left\{\begin{array}{l}
\nabla^{2} \phi^{\prime}=-\frac{g K \beta}{v} \frac{\partial}{\partial x^{\prime}} T_{2}^{\prime} \\
(\rho c)_{\mathrm{p}} \frac{\partial T_{2}^{\prime}}{\partial t^{\prime}}+(\rho c)_{\mathrm{f}} V^{\prime} \cdot \nabla T_{2}^{\prime}=\lambda_{\mathrm{p}} \nabla^{2} T_{2}^{\prime} \\
\varepsilon \frac{\partial C^{\prime}}{\partial t^{\prime}}+\vec{V}^{\prime} \cdot \nabla C^{\prime}=\nabla \cdot\left(D^{*} \nabla C^{\prime}+C^{\prime}\left(1-C^{\prime}\right) D_{T}^{*} \nabla T_{2}^{\prime}\right)
\end{array}\right.
$$


where $V^{\prime}$ is the Darcy velocity, $T_{2}^{\prime}$ the temperature inside the porous bulk, $g$ the gravitational acceleration, $v$ the kinematic viscosity, $(\rho c)_{\mathrm{p}}$ and $(\rho c)_{\mathrm{f}}$ are the respective heat capacities of the saturated porous medium and the fluid, $\lambda_{\mathrm{p}}$ is the effective thermal conductivity of the saturated porous medium and $\phi^{\prime}$ is the stream function. As usual, the equation of continuity is satisfied by introducing the stream function according to $u^{\prime}=\partial \phi^{\prime} / \partial y^{\prime}$ and $v^{\prime}=-\partial \phi^{\prime} / \partial x^{\prime}$

For the two plates bounding the porous medium, we have

$$
\begin{aligned}
& (\rho c)_{\mathrm{s}} \frac{\partial T_{1}^{\prime}}{\partial t^{\prime}}=\lambda_{\mathrm{s}} \nabla^{2} T_{1}^{\prime}, \\
& (\rho c)_{\mathrm{s}} \frac{\partial T_{3}^{\prime}}{\partial t^{\prime}}=\lambda_{\mathrm{s}} \nabla^{2} T_{3}^{\prime},
\end{aligned}
$$

where $T_{1}^{\prime}$ and $T_{3}^{\prime}$ are the temperatures inside the lower and upper plates, respectively, and $(\rho c)_{\mathrm{s}}$ and $\lambda_{\mathrm{s}}$ are the heat capacity and the thermal conductivity of the solid material. We have assumed that bounding plates are made from the same material and are of identical thicknesses.

The boundary conditions applied to the horizontal boundaries of the system are uniform fluxes of heat per unit area, $q^{\prime}$. It is assumed that the vertical walls of the cavity are thermally well insulated and impermeable.

We assume that there is little variation in the term $C(1-C)$ of the equation of conservation of species, so we can replace it by $C_{0}\left(1-C_{0}\right)$, where $C_{0}$ is the initial mass fraction.

The reference scales are $H$ for the length, $a / H$ for the velocity with $a=\lambda_{\mathrm{p}} /(\rho c)_{\mathrm{f}}$ ( $a$ is the effective thermal diffusivity), $\varphi H^{2} / a$ for the time (with $\varphi=(\rho c)_{\mathrm{p}} /(\rho c)_{\mathrm{f}}$, the heat capacity ratio), $\Delta T=q^{\prime} H / \lambda_{\mathrm{p}}$ for the temperature, $\Delta C=-\Delta T C_{0}\left(1-C_{0}\right)\left(D_{\mathrm{T}}^{*} / D^{*}\right)$ for the mass fraction, where $D_{\mathrm{T}}^{*}$ and $D^{*}$ are, respectively, the thermodiffusion and mass-diffusion coefficients of the denser component.

The dimensionless mathematical formulation of the problem is given by

$$
\left\{\begin{array}{l}
\nabla^{2} \phi=-R a \frac{\partial}{\partial x}\left(T_{2}+\psi C\right) \\
\frac{\partial T_{2}}{\partial t}+\vec{V} \cdot \vec{\nabla} T_{2}=\nabla^{2} T_{2} \\
\varepsilon \frac{\partial C}{\partial t}+\vec{V} \cdot \vec{\nabla} C=\frac{1}{L e}\left(\nabla^{2} C-\nabla^{2} T_{2}\right) \\
\frac{\partial T_{1}}{\partial t}=\alpha \nabla^{2} T_{1} \\
\frac{\partial T_{3}}{\partial t}=\alpha \nabla^{2} T_{3}
\end{array}\right.
$$

The problem under consideration depends on eight non-dimensional parameters: the thermal Rayleigh number $R a=\left(g \beta_{\mathrm{T}} K \Delta T H\right) /(a v)$ ( $K$ is the permeability of the porous medium); the separation ratio $\psi=-\left(\beta_{\mathrm{C}} / \beta_{\mathrm{T}}\right)\left(D_{\mathrm{T}}^{*} / D^{*}\right) C_{0}\left(1-C_{0}\right)$; the Lewis number $L e=$ $a / D^{*}$; the normalized porosity $\varepsilon=\varepsilon^{*} / \varphi$; the thermal diffusivity ratio $\alpha=a_{\mathrm{s}} / a_{\mathrm{p}}$, where $a_{s}=\lambda_{\mathrm{s}} /(\rho c)_{\mathrm{s}}$ and $a_{\mathrm{p}}=\lambda_{\mathrm{p}} /(\rho c)_{\mathrm{p}}$ are, respectively, the thermal diffusivity of the metal plates and porous medium; the thermal conductivity ratio $d=\lambda_{\mathrm{s}} / \lambda_{\mathrm{p}}$; the aspect ratio $\delta=h / H$; and the aspect ratio of the porous cell $A=L / H$. In the present study, the intensity of the thermal buoyancy forces is expressed solely in terms of the parameter $R a$. 
The corresponding dimensionless boundary conditions are:

$$
\begin{aligned}
& \text { for } \quad y=-\delta, \quad \frac{\partial T_{1}}{\partial y}=-\frac{\lambda_{\mathrm{p}}}{\lambda_{\mathrm{s}}}=\frac{-1}{d} ; \\
& \text { for } \quad y=0, \quad \frac{\partial T_{1}}{\partial y}=\frac{1}{d} \frac{\partial T_{2}}{\partial y}, \quad T_{1}=T_{2}, \quad \phi=0, \quad \frac{\partial C}{\partial y}-\frac{\partial T_{2}}{\partial y}=0 ; \\
& \text { for } \quad y=1, \quad \frac{\partial T_{3}}{\partial y}=\frac{1}{d} \frac{\partial T_{2}}{\partial y}, \quad T_{2}=T_{3}, \quad \phi=0, \quad \frac{\partial C}{\partial y}-\frac{\partial T_{2}}{\partial y}=0 ; \\
& \text { for } \quad y=1+\delta, \quad \frac{\partial T_{3}}{\partial y}=\frac{-1}{d}
\end{aligned}
$$

\section{Linear stability of the equilibrium solution}

\subsection{Linear stability of the equilibrium solution in an infinite horizontal cell}

It is straightforward to show that there exists an equilibrium solution characterized by

$$
\begin{aligned}
& \vec{V}=0, \quad T_{1,0}=-y / d+T_{\mathrm{a}}, \quad T_{2,0}=T_{\mathrm{a}}-y, \\
& T_{3,0}=(1-y) / d+T_{\mathrm{a}}-1, \quad C_{0}=1 / 2-y,
\end{aligned}
$$

where $T_{\mathrm{a}}$ is an arbitrary constant temperature. In order to analyze the stability of this equilibrium solution, we first introduce the perturbations of stream function, of temperatures, namely $\theta_{1}, \theta_{2}$ and $\theta_{3}$, and of the mass fraction $c$. We assume that the perturbations $\left(\phi, \theta_{1}, \theta_{2}, \theta_{3}, c\right)$ are of small amplitude, and for simplicity, we introduce the new function $\eta=c-\theta_{2}$, so that (4) leads to

$$
\left\{\begin{array}{l}
\nabla^{2} \phi+R a \frac{\partial}{\partial x}\left[\theta_{2}(1+\psi)+\psi \eta\right]=0 \\
\frac{\partial \theta_{2}}{\partial t}-\nabla^{2} \theta_{2}=-\frac{\partial \phi}{\partial x} \\
\varepsilon L e \frac{\partial\left(\eta+\theta_{2}\right)}{\partial t}-\nabla^{2}(\eta)=-\frac{\partial \phi}{\partial x} L e \\
\frac{\partial \theta_{1}}{\partial t}-\alpha \nabla^{2} \theta_{1}=0 \\
\frac{\partial \theta_{3}}{\partial t}-\alpha \nabla^{2} \theta_{3}=0
\end{array}\right.
$$

The perturbation quantities are chosen as follows:

$$
\left(\phi, \theta_{1}, \theta_{2}, \theta_{3}, \eta\right)=\left(\tilde{\phi}, \tilde{\theta}_{1}, \tilde{\theta}_{2}, \tilde{\theta}_{3}, \tilde{\eta}\right)(y) \exp (I k x+\sigma t)+\text { c.c. },
$$

where $k$ is the wave number in the horizontal $(x)$ direction, $I^{2}=-1$, and $\sigma$ is the temporal exponential growth rate of perturbation.

3.1.1. Stationary transition In the first part, we focus on steady bifurcation. When the expansions (8) are introduced in (7) and $\sigma=0$ (steady bifurcation), we obtain the following 
linearized equations:

$$
\left\{\begin{array}{l}
\left(D^{2}-k^{2}\right) \tilde{\phi}+\operatorname{RaIk}\left[(1+\psi) \tilde{\theta}_{2}+\psi \tilde{\eta}\right]=0, \\
\left(D^{2}-k^{2}\right) \tilde{\theta}_{2}-I k \tilde{\phi}=0 \\
\left(D^{2}-k^{2}\right) \tilde{\eta}-I k L e \tilde{\phi}=0 \\
\left(D^{2}-k^{2}\right) \tilde{\theta}_{1}=0 \\
\left(D^{2}-k^{2}\right) \tilde{\theta}_{3}=0
\end{array}\right.
$$

where $D=\mathrm{d} / \mathrm{d} y$.

Removing $\tilde{\phi}$ from system (9), the following linear system is obtained:

$$
\left\{\begin{array}{l}
\left(D^{2}-k^{2}\right)^{2} \tilde{\theta}_{2}-R a k^{2}[1+\psi+\psi L e] \tilde{\theta}_{2}=R a k^{2} \psi[A \sinh (k y)+B \cosh (k y)], \\
\left(D^{2}-k^{2}\right) \tilde{\theta}_{1}=0, \\
\left(D^{2}-k^{2}\right) \tilde{\theta}_{3}=0,
\end{array}\right.
$$

where $A$ and $B$ verify

$$
\begin{aligned}
& L e\left(\mathrm{~d} \tilde{\theta}_{2} / \mathrm{d} y\right)+k A=0, \quad \text { for } y=0 ; \\
& L e\left(\mathrm{~d} \tilde{\theta}_{2} / \mathrm{d} y\right)+k A \cosh (k)+k B \sinh (k)=0, \quad \text { for } y=1,
\end{aligned}
$$

with the associated boundary conditions:

$$
\begin{aligned}
& \text { for } y=-\delta, \quad \frac{\partial \tilde{\theta}_{1}}{\partial y}=0 \\
& \text { for } y=0, \quad \frac{\partial \tilde{\theta}_{1}}{\partial y}=\frac{1}{d} \frac{\partial \tilde{\theta}_{2}}{\partial y}, \quad \tilde{\theta}_{1}=\tilde{\theta}_{2}, \quad\left(D^{2}-k^{2}\right) \tilde{\theta}_{2}=0, \frac{\partial \tilde{\eta}}{\partial y}=0 \text {; } \\
& \text { for } y=1, \quad \frac{\partial \tilde{\theta}_{3}}{\partial y}=\frac{1}{d} \frac{\partial \tilde{\theta}_{2}}{\partial y}, \quad \tilde{\theta}_{2}=\tilde{\theta}_{3}, \quad\left(D^{2}-k^{2}\right) \tilde{\theta}_{2}=0, \frac{\partial \tilde{\eta}}{\partial y}=0 \text {; } \\
& \text { for } y=1+\delta, \quad \frac{\partial \tilde{\theta}_{3}}{\partial y}=0 .
\end{aligned}
$$

The general solution of the fourth-order ordinary differential equation (10) is given as a combination of four particular independent functions whose expression depends on the sign of $R a(1+\psi+\psi L e)$. These considerations permit us to consider four regions of the plane $(R a, \psi)$. The solution of the first equation of system (10) is the sum of the general solution of the homogeneous associated equation, $\tilde{\theta}_{2 \mathrm{hs}}$, and a particular solution, $\tilde{\theta}_{2 \mathrm{ps}}$,

$$
\tilde{\theta}_{2 \mathrm{ps}}=\frac{-\psi[A \sinh (k y)+B \cosh (k y)]}{1+\psi+\psi L e} .
$$

The solution $\tilde{\theta}_{2 \mathrm{hs}}$ is sought under $\mathrm{e}^{\mathrm{ry}}$ form, which leads to the following characteristic equation:

$$
\left(r^{2}-k^{2}\right)^{2}-\operatorname{Rak}^{2}(1+\psi+\psi L e)=0 .
$$

It is almost an algebraic equation of the fourth degree. The real or complex nature of the four roots of this equation depends on the sign of $R a(1+\psi+\psi L e)$.

(a) The case $R a(1+\psi+\psi L e)>0$.

In this last case, we find $\tilde{\theta}_{2 \mathrm{hs}}=C \cosh \left(R_{1} y\right)+D \sinh \left(R_{1} y\right)+E \cos \left(R_{2} y\right)+F \sin \left(R_{2} y\right)$. 
The general solution of system (10) associated with this case is

$$
\begin{gathered}
\tilde{\theta}_{2}(y)=C \cosh \left(R_{1} y\right)+D \sinh \left(R_{1} y\right)+E \cos \left(R_{2} y\right)+F \sin \left(R_{2} y\right) \\
-\frac{\psi[A \sinh (k y)+B \cosh (k y)]}{(1+\psi+\psi L e)} ; \\
\tilde{\theta}_{1}(y)=G \cosh (k y)+H \sinh (k y) ; \quad \tilde{\theta}_{3}(y)=M \cosh (k y)+N \sinh (k y),
\end{gathered}
$$

where

$$
R_{1}=\sqrt{(\sqrt{R a(1+\psi+\psi L e)}+k) k} \quad \text { and } \quad R_{2}=\sqrt{(\sqrt{R a(1+\psi+\psi L e)}-k) k}
$$

The solution obtained depends on the ten arbitrary constants A, B, C, D, E, F G, H, M and $\mathrm{N}$.

When we assume that this general solution satisfies the ten boundary conditions given in equations (11) and (12), we obtain ten homogeneous linear algebraic equations and ten unknowns corresponding to the ten constants. This system has a non-trivial solution if the associated matrix determinant, $\operatorname{det}(\operatorname{Ra}(k), k, \delta, d, \psi, L e)$, is equal to zero. The expression for this determinant was obtained using the Maple algebra code. Once we calculated the determinant, we obtained the dispersion relation function of the Rayleigh number, the wave number $k$, the aspect ratio $\delta$, the thermal conductivity ratio $d$, the Lewis number $L e$ and the separation ratio $\psi$. The expression for the dispersion equation is lengthy and complicated, so it will be discussed later for the case of long-wave disturbances in the case (d).

(b) The case $R a(1+\psi+\psi L e)<0$.

We proceed in the same manner as before. We calculate first the four roots of the characteristic equation, which are complex (the complex part, its conjugate and their opposites). The general solution of the differential equation of fourth order without a second member is of the form

$$
\tilde{\theta}_{2}(y)=\mathrm{e}^{\alpha y}[A \sin (\beta y)+B \cos (\beta y)]+\mathrm{e}^{-\alpha y}[C \sin (\beta y)+D \cos (\beta y)]
$$

with

$$
\begin{aligned}
& \alpha=\sqrt{\frac{k^{2}}{2}+\frac{k}{2} \sqrt{R a_{m}^{2}-k^{2}}}, \\
& \beta=\sqrt{-\frac{k^{2}}{2}+\frac{k}{2} \sqrt{R a_{m}^{2}-k^{2}}}, \\
& R a_{m}=\sqrt{-R a(1+\psi+\text { Le })} .
\end{aligned}
$$

In this case, the determinant is written as

$$
\operatorname{det}(R a(k), k, \delta, d, \psi, L e)=\frac{k^{4}}{8} \frac{\sqrt{2 k^{2}+2 k \sqrt{R a_{m}^{2}-k^{2}}}}{(1+\psi+\psi L e)^{2}} G(R a, \delta, d, \psi, L e) .
$$

From this relation, we directly determine the critical values of Rayleigh number and wave number, $R a_{\mathrm{c}}$ and $k_{\mathrm{c}}$. 
(c) The case $1+\psi+\psi L e=0$.

For the particular case $1+\psi+\psi L e=0$, the system of equation (10) is solved analytically, using the Maple algebra code. We obtain two possible dispersion equations corresponding to the first and second bifurcations,

$$
R a_{1}=g_{1}(d, \delta, k) \frac{(L e+1)}{L e} \quad \text { and } \quad R a_{2}=g_{2}(d, \delta, k) \frac{(L e+1)}{L e} .
$$

where

$g_{1}(d, \delta, k)=\frac{8 k^{2}\left(1-\mathrm{e}^{2 k}\right)\left[(1-d)\left(\mathrm{e}^{k}+\mathrm{e}^{2 k \delta}\right)+(1+d)\left(1+\mathrm{e}^{k(1+2 \delta)}\right)\right]}{d\left(\mathrm{e}^{2 k \delta}-1\right)\left[2 k^{2} \mathrm{e}^{2 k}-2 k^{2} \mathrm{e}^{k}-2 k \mathrm{e}^{k}-2 k \mathrm{e}^{2 k}+\mathrm{e}^{3 k}+\mathrm{e}^{2 k}-\mathrm{e}^{k}-1\right]}$,

$g_{2}(d, \delta, k)=\frac{8 k^{2}\left(1-\mathrm{e}^{2 k}\right)\left[(1-d)\left(\mathrm{e}^{k}-\mathrm{e}^{2 k \delta}\right)+(1+d)\left(\mathrm{e}^{k(1+2 \delta)}-1\right)\right]}{d\left(\mathrm{e}^{2 k \delta}-1\right)\left[2 k^{2} \mathrm{e}^{2 k}-2 k^{2} \mathrm{e}^{k}-2 k \mathrm{e}^{k}-2 k \mathrm{e}^{2 k}+\mathrm{e}^{3 k}+\mathrm{e}^{2 k}-\mathrm{e}^{k}-1\right]}$.

Using the experimental values taken by Platten et al (2003), the thermophysical properties of glass spheres and copper: $(d, \delta, L e)=(28.4,3,232)$, we deduce the critical values of Rayleigh number and wave number associated with the first and second bifurcations,

$R a_{\mathrm{c} 1}=-12 \frac{(L e+1)}{L e}, \quad k_{\mathrm{c} 1}=0, \quad R a_{\mathrm{c} 2}=-64.30 \frac{(L e+1)}{L e}, \quad k_{\mathrm{c} 2}=3.40$.

All analytical results and physical interpretations found from the cases (a), (b) and (c) are summarized in table 1 and figures 3 and 4 . The results obtained are in very good agreement with those obtained using the spectral Tau method. The disturbances are developed in terms of polynomial functions verifying all the boundaries conditions except those along the inner plates:

$$
\begin{aligned}
& \tilde{\theta}_{1}(y)=a_{1}+a_{2}\left[y+\frac{y^{2}}{2 \delta}\right]+\sum_{n=1}^{N-2} a_{n+2} y^{n}(y+\delta)^{2} ; \quad \tilde{\theta}_{2}(y)=\sum_{n=1}^{N} b_{n} y^{n-1} ; \\
& \tilde{\theta}_{3}(y)=c_{1}+c_{2}\left[y-\frac{y^{2}}{2(1+\delta)}\right]+\sum_{n=1}^{N-2} c_{n+2} y^{n}(y-1-\delta)^{2} ; \\
& \tilde{\phi}(y)=\sum_{n=1}^{N} f_{n}(1-y) y^{n} ; \quad \tilde{\eta}(y)=d_{1}+d_{2}\left(y^{2}-\frac{2}{3} y^{3}\right)+\sum_{n=1}^{N-2} d_{n+2} y^{n+1}(1-y)^{2} .
\end{aligned}
$$

The Tau spectral method used is similar to the Galerkin method. The test functions are equal to the trial functions but some of the trial functions employed do not satisfy all the boundary conditions. The trial functions used for $\tilde{\phi}$ and $\tilde{\eta}$ verify all the boundary conditions, so the residue $R_{\phi}$ associated with the first equation of system (7) is multiplied by $(1-y) y^{i}$ with $i$ varying from 1 to $N, \forall i=1 \ldots N, \quad \int_{0}^{1} R_{\phi}(1-y) y^{i} \mathrm{~d} y=0$, leading to $N$ algebraic equations. A similar procedure is used for the residue $R_{\eta}$ associated, respectively, with the third equation of system (7). The coupling boundary conditions for $\tilde{\theta}_{2}$ with $\tilde{\theta}_{1}$ and $\tilde{\theta}_{3}$ lead us to the choice of the trial function $y^{i}$, members of a complete set of functions for $\tilde{\theta}_{2}$. The residue $R_{\theta_{2}}$ associated with the second equation of system (7) is multiplied by $y^{i}$ with $i$ varying from 0 to $N-1, \forall i=0 \ldots N-2, \quad \int_{0}^{1} R_{\theta_{2}} y^{i} \mathrm{~d} y=0$. The trial functions used for $\tilde{\theta}_{1}$ and $\tilde{\theta}_{3}$ satisfy, respectively, only the boundary condition on $y=-\delta$ and on $y=1+\delta$. For the fourth and fifth equations of system (7), the associated residues $R_{\theta_{1}}$ and $R_{\theta_{3}}$ are multiplied by the associated test function and integrated, respectively, on the segments $[-\delta, 0]$ and $[1,1+\delta]$, 
Table 1. Comparison between the values of critical Rayleigh number $R a_{\mathrm{cs}}$ and the critical wave number $k_{\mathrm{cs}}$ obtained by the exact solution and spectral Tau methods at fifth order.

\begin{tabular}{|c|c|c|c|c|c|}
\hline & & \multicolumn{2}{|c|}{$d=5, \delta=1, L e=5$} & \multicolumn{2}{|c|}{$d=28.4, \delta=3, L e=232$} \\
\hline & & $R a_{\mathrm{cs}}$ & $k_{\mathrm{cs}}$ & $R a_{\mathrm{cs}}$ & $k_{\mathrm{cs}}$ \\
\hline \multirow[t]{2}{*}{$\Psi=-0.1$} & Exact & 147.300 & 4.610 & $\infty$ & - \\
\hline & Tau & 147.290 & 4.610 & $\infty$ & - \\
\hline \multirow[t]{2}{*}{$\Psi=-0.05$} & Exact & 61.753 & 3.495 & $\infty$ & - \\
\hline & Tau & 61.752 & 3.495 & $\infty$ & - \\
\hline \multirow{2}{*}{$\Psi=-0.02$} & Exact & 43.028 & 3.038 & $\infty$ & - \\
\hline & Tau & 43.027 & 3.038 & $\infty$ & - \\
\hline \multirow[t]{2}{*}{$\Psi=0.00$} & Exact & 35.175 & 2.786 & 38.169 & 3.073 \\
\hline & Tau & 35.174 & 2.786 & 38.169 & 3.073 \\
\hline \multirow[t]{2}{*}{$\Psi=0.02$} & Exact & 29.439 & 2.564 & 2.583 & 0.00 \\
\hline & Tau & 29.440 & 2.564 & 2.583 & 0.00 \\
\hline \multirow[t]{2}{*}{$\Psi=0.10$} & Exact & 16.946 & 1.875 & 0.517 & 0.00 \\
\hline & Tau & 16.945 & 1.875 & 0.517 & 0.00 \\
\hline \multirow[t]{2}{*}{$\Psi=0.15$} & Exact & 13.064 & 1.538 & 0.345 & 0.00 \\
\hline & Tau & 13.064 & 1.538 & 0.345 & 0.00 \\
\hline \multirow[t]{2}{*}{$\Psi=0.20$} & Exact & 10.504 & 1.233 & 0.258 & 0.00 \\
\hline & Tau & 10.504 & 1.233 & 0.258 & 0.00 \\
\hline
\end{tabular}

leading to $N-2$ algebraic equations. A supplementary set of four equations is used to apply the boundary conditions which are not verified by the trial functions:

$$
\begin{aligned}
& \tilde{\theta}_{1}(0)=\tilde{\theta}_{2}(0), \quad \tilde{\theta}_{2}(1)=\tilde{\theta}_{3}(1), \quad \frac{\partial \tilde{\theta}_{1}(0)}{\partial y}=\frac{1}{d} \frac{\partial \tilde{\theta}_{2}(0)}{\partial y} \text { and } \\
& \frac{\partial \tilde{\theta}_{2}(1)}{\partial y}=\frac{1}{d} \frac{\partial \tilde{\theta}_{3}(1)}{\partial y},
\end{aligned}
$$

leading to four algebraic equations. This yields $5 N$ algebraic equations for $5 N$ unknown constants.

Table 1 reports the critical values of $R a_{\mathrm{cs}}$ and $k_{\mathrm{cs}}$ obtained with the exact solution and spectral Tau methods, for the first bifurcation by fixing the values of $L e, d, \delta$ and for different values of $\psi$. In figures $2(\mathrm{a})$ and (b), we present the stability diagrams $\operatorname{Ra}_{\mathrm{cs}}=f(\psi)$ and the $R a_{0}$ values associated with $k=0$, obtained for $(L e, d, \delta)=(5,5,1)$ and $(L e, d, \delta)=$ $(232,28.4,3)$. The two sets of parameters correspond, respectively, to the binary gas and the binary solution. For large values of the Lewis number, the critical Rayleigh number decreases significantly depending on.

In figures $3(\mathrm{a})$ and (b), the stability diagram $k_{\mathrm{cs}}=f(\psi)$ for $(L e, d, \delta)=(5,5,1)$ and $(L e, d, \delta)=(232,28.4,3)$ clearly shows the variation of the critical wave number versus the separation ratio for stationary bifurcation.

An $R a_{\mathrm{cs}}=R a_{0}$ and $k_{\mathrm{cs}}=0$ for $R a<0 \forall \psi$ and $R a>0$ only for $\psi \geqslant \psi_{\text {uni. }}$.

For $\psi \in\left[\psi_{H}, \psi_{\text {uni }}\right]$, we have $k_{\mathrm{cs}} \neq 0$. The expression for $\psi_{H}, \psi_{\text {uni }}$ and $R a_{0}$ will be given in the case (d).

For $\psi>0$, the denser component moves toward the cooler wall. The pure doublediffusive solution is then infinitely linearly stable when the horizontal layer is heated from above, while it loses its stability for critical Rayleigh numbers smaller than $4 \pi^{2}$ which corresponds to the critical Rayleigh number for the pure thermal problem and for $R a>0$. For $\psi<0$, the denser component moves toward the warmer wall. For $R a<0$, the equilibrium 

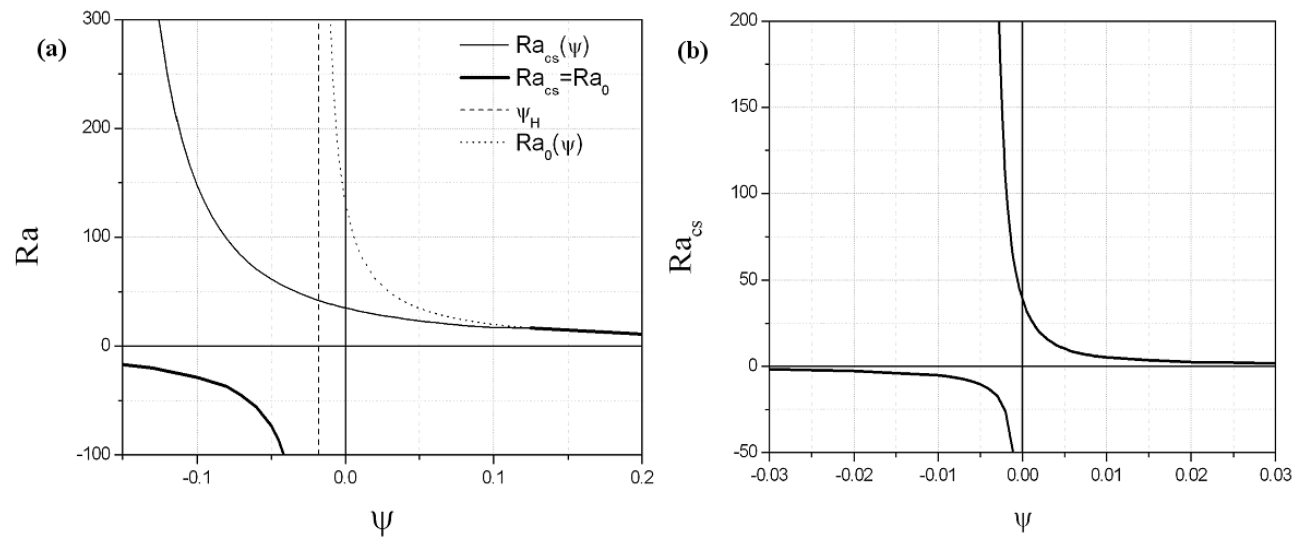

Figure 2. (a, b) Critical Rayleigh number at the onset of convection versus separation ratio for (a) $L e=5, \delta=1, d=5$ and (b) $L e=232, \delta=3, d=28.4$. Dashed lines: $\psi_{\mathrm{H}}=$ $-1 /(L e(1+2 d \delta)+1)$ and $R a_{0}=12(1+2 d \delta) /[1+\psi(2 d \delta L e+L e+1)]$.
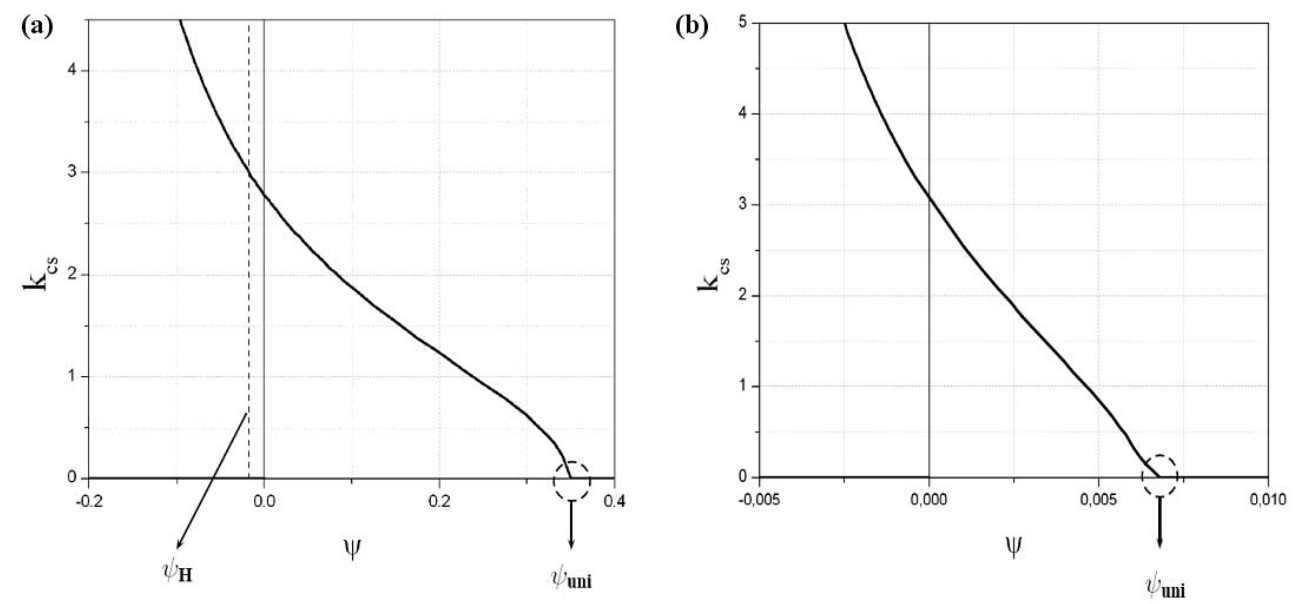

Figure 3. Critical wave number at the onset of convection versus separation ratio for (a) $L e=5$, $\delta=1, d=5$ and (b) $L e=232, \delta=3, d=28.4$. Dashed line: $\psi_{\mathrm{H}}=-1 /(\operatorname{Le}(1+2 d \delta)+1)$.

solution is not linearly stable, unlike the pure thermal problem, so we can expect that $R a_{\mathrm{cs}} \rightarrow-\infty$ when $\psi \rightarrow 0^{-}$. This result was confirmed by our direct simulations. For $R a>0$, $(1+\psi+\psi L e)>0$ and $\psi<0$, the downward migration of the denser component leads to an increase of the critical Rayleigh number $\left(R a_{\mathrm{cs}}>4 \pi^{2}\right)$. For $R a>0$ and $(1+\psi+\psi L e)<0$, we do not obtain the transition to the stationary convective regime.

(d) The limiting case of long-wave disturbances $k \approx 0$.

The case of long-wave disturbances (for which the wave number, $k$, is close to zero) may be studied analytically using the dispersion relation, by developing a regular perturbation expansion with $k$ as a small parameter. In our case, we expand the function $F$ in the vicinity of $k=0$ using Maple software; then we obtain

$$
\operatorname{det}(R a(k), k, \delta, d, \psi, L e)=\frac{R a k^{6}}{1+\psi+\psi L e} F(R a, \delta, d, \psi, L e) .
$$


The singular case $1+\psi+\psi L e=0$ was studied analytically in the case (c). From this dispersion equation, we determine the critical wave number and the critical Rayleigh number. For this purpose, we fix the values of $d, \delta$ and the Lewis number Le. For every value of $\psi$ fixed, we look for the value of $k=k_{\mathrm{cs}}$, which minimizes the Rayleigh number to obtain the associated value, $R a_{\text {cs }}$.

We develop the function $F$ in the vicinity of $k=0$, which gives

$F(R a, \delta, d, \psi, L e)=F_{1}(R a, \delta, d, \psi, L e)+k^{2} F_{2}(R a, \delta, d, \psi, L e)+0\left(k^{4}\right)$.

The function $F_{1}(R a, \delta, d, \psi, L e)$ is equal to zero for

$$
R a_{0}=\frac{12(1+2 d \delta)}{1+\psi(2 L e d \delta+L e+1)} .
$$

This value of $R a_{0}$ corresponds to the onset of the unicellular flow associated with $k=0 . R a_{0}$ does not necessarily correspond to a critical Rayleigh number of the problem considered, as shown in figure 2(a). By replacing $R a$ by $R a_{0}$ in the expression of $F_{2}$, the equation $F_{2}(R a, \delta, d, \psi, L e)=0$, after simplification, leads to a relationship linking the various physical parameters $(L e, \delta, d, \psi)$ which is written as

$$
10(1+2 d \delta)^{2} \psi L e+\left[10-d \delta(1+\psi)\left(70 \delta^{3}+51 d \delta-12\right)\right]=0
$$

The solution of this equation (20) with regard to $\psi$ leads to

$$
\psi_{\text {uni }}=\frac{1}{\frac{10(1+2 d \delta)^{2}}{51 d^{2} \delta^{2}+70 d \delta^{3}-10-12 d \delta}-1} .
$$

Let us look at the conditions under which $k=0$ and

$$
R a_{0}=\frac{12(1+2 d \delta)}{1+\psi(2 L e d \delta+L e+1)}
$$

correspond to the critical parameters $k_{\mathrm{cs}}=0, R a_{\mathrm{cs}}=R a_{0}$. For this purpose, we distinguish two cases, $R a>0$ and $R a<0$ :

For $R a>0$, we have to show that $\frac{\mathrm{d} R a}{\mathrm{~d} k^{2}} \geqslant 0$, and for $R a<0$, we have to show that $\frac{\mathrm{d} R a}{\mathrm{~d} k^{2}} \leqslant 0$ in the neighborhood of $k=0$.

When $k$ tends to zero, we verify that

$$
\left(\frac{\mathrm{d} R a}{\mathrm{~d} k^{2}}\right)_{k=0}=-F_{2}\left(R a_{0}, \delta, d, \psi, L e\right)
$$

and we deduce that

$$
\left(\frac{\mathrm{d} R a}{\mathrm{~d} k^{2}}\right)_{k=0}=\frac{4\left[10(1+2 d \delta)^{2} \psi L e+(1+\psi)\left(12 d \delta+10-70 d \delta^{3}-51 d^{2} \delta^{2}\right)\right]}{35(\psi[L e(2 d \delta+1)+1]+1)^{2}} .
$$
thus

The sign of $\left(\frac{\mathrm{d} R a}{\mathrm{~d} k^{2}}\right)$ is the same as the sign of the numerator $N_{\text {num }}$ of equation (23), and

$$
\left(\frac{\mathrm{d} R a}{\mathrm{~d} k^{2}}\right)_{k=0} \rightarrow \infty \quad \text { for } \quad \psi=\psi_{H}=\frac{-1}{1+L e(1+2 d \delta)} .
$$

We can make three remarks concerning the critical Rayleigh number found in our study:

Remark $1 \delta \rightarrow 0$ means that the thickness of the walls delimiting the porous medium tends to zero. This configuration corresponds to a constant heat flux directly imposed on the porous 
layer, as studied previously by Bahloul et al. (2003). We obtain the same results as those obtained by these authors when $\delta \rightarrow 0, R a_{\mathrm{c}}=12 /(1+\psi+\psi L e)$.

Remark $2 d \rightarrow \infty$ means that the walls delimiting the porous medium are infinitely more conductive than the porous layer saturated by with binary fluid. This configuration is similar to a porous cell maintained at a constant temperature difference. This configuration has been studied by Schöpf (1992) and Charrier-Mojtabi et al (2007). For $d \rightarrow \infty$, the relation giving $R a_{\mathrm{c}}$ leads to $R a_{\mathrm{c}}=12 / \psi L e$, a result obtained previously by those authors. Regarding convection in a binary fluid, Knobloch and Moore (1988) obtained analytically a similar result: $R a_{\mathrm{c}}=720 / \psi$ Le.

Remark 3 When we take into account the effect of the walls delimiting the porous medium, and for $\psi=0$ (natural convection), the relation giving $R a_{\mathrm{c}}$ leads to $R a_{\mathrm{c}}=12(1+2 d \delta)$, a result obtained recently by Mojtabi and Rees (2011).

3.1.2. Oscillatory instability In order to study the stability of the equilibrium solution, we introduce and expand the perturbations using equation (8). When we replace the disturbances by their developed form in system (7), and take $\sigma=I \omega$ (oscillatory bifurcation), we find the second-order differential system:

$$
\left\{\begin{array}{l}
\left(D^{2}-k^{2}\right) \phi+I k R a\left[(1+\psi) \theta_{2}+\psi \eta\right]=0, \\
\left(D^{2}-k^{2}-I \omega\right) \theta_{2}-I k \phi=0, \\
\left(D^{2}-k^{2}-I L e \varepsilon \omega\right) \eta-I L e \varepsilon \omega \theta_{2}-I k \phi=0, \\
\alpha\left(D^{2}-k^{2}\right) \theta_{1}-I \omega \theta_{1}=0, \\
\alpha\left(D^{2}-k^{2}\right) \theta_{3}-I \omega \theta_{3}=0 .
\end{array}\right.
$$

The corresponding boundary conditions are given by (12).

To solve this linear differential system, we use the spectral Tau method and the same disturbances given in equation (16) with no restriction on the range of parameters $R a$, $\psi$ and $L e$. Replacing the disturbances by their developments in system (24) leads to a homogeneous linear algebraic system. This system has a non-trivial solution if the associated matrix determinant is equal to zero. In this study, the determinant is a complex:

$\operatorname{det}(A)=R(R a, k, \omega, \varepsilon, L e, \psi, d, \delta)+I Q(R a, k, \omega, \varepsilon, L e, \psi, d, \delta)=0$,

where $R$ and $Q$ are real polynomials. To calculate the value of the critical Rayleigh number corresponding to a Hopf bifurcation, we proceed as follows, using the computer software code Maple:

We first fix the particular values of $\psi, \varepsilon, d, \delta$ and $L e$. We resolve the following algebraic system, with two unknowns $(R a, \omega)$ and a parameter $k$ :

$$
\left\{\begin{array}{l}
R(R a, k, \omega)=0, \\
Q(R a, k, \omega)=0 .
\end{array}\right.
$$

When we solve this system, we obtain the real roots of indices $j: R a_{j}=f_{j}(k)$ and $\omega_{j}=h_{j}(k)$. We can then look for the minimum value of $R a$ according to $k$ and obtain the critical parameters $R a_{j c}=f_{j}\left(k_{c}\right)$ and $\omega_{j c}=h_{j}\left(k_{c}\right)$.

When we set $\omega=0$ in the expression for the determinant $\operatorname{det}(A)$, we verify that the critical Rayleigh numbers obtained correspond to the stationary transition. When $\omega$ is different from 0 , we are led to determine the critical Rayleigh numbers corresponding to the oscillatory 

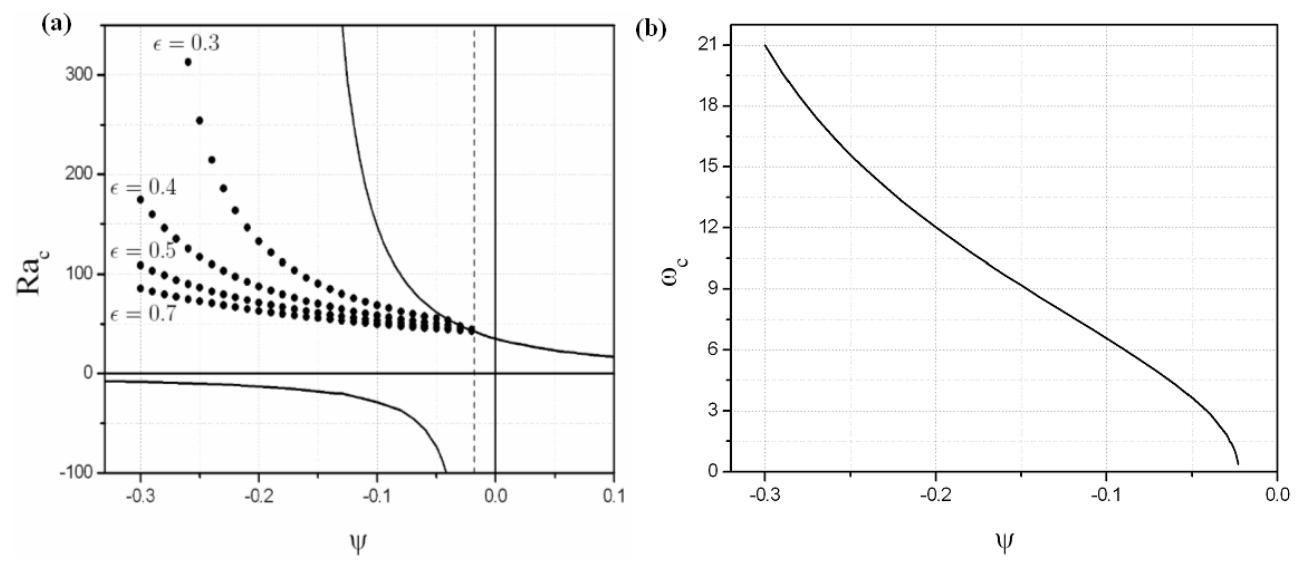

Figure 4. (a) Stability diagram for different normalized porosity values $(\varepsilon=0.3,0.4,0.5,0.7)$ (the spectral Tau method at fifth order) and for $L e=5, \delta=1, d=5$. Solid line, stationary bifurcation; dotted line, Hopf bifurcation; dashed line, $\psi_{\mathrm{H}}=-1 /(\operatorname{Le}(1+2 d \delta)+1)$. (b) Critical Hopf frequency number versus separation ratio at fifth order for $\varepsilon=0.4, L e=5, \delta=1$ and $d=5$.

bifurcation. We were able to determine these critical parameters using the Maple software code.

In the case of a cell heated from below, for $\psi<0$, the heaviest constituent migrates toward the hot wall and a stabilizing effect occurs.

In figure 4(a), we show that, for values of $\psi$ less than $\psi_{\mathrm{H}}=-1 /(\operatorname{Le}(1+2 d \delta)+1)$, the primary bifurcation is a Hopf bifurcation. Using the spectral Tau method to fifth order with $d, \delta, L e$ fixed and for different values of $\varepsilon$ and $\psi$, we determined the critical Rayleigh number $R a_{\text {co }}$, the critical Hopf frequency $\omega_{\text {co }}$ and the associated critical wave number $k_{\text {co }}$ (figure 4(b)). The marginal stability curves associated with the Hopf bifurcations are slightly below the curve of marginal stability associated with the stationary bifurcation regardless of the value of the normalized porosity considered.

\section{Conclusion}

The critical Rayleigh number at the onset of the Soret-driven convection was determined using a linear stability analysis in the case of an infinite horizontal cell. The influence of a negative or positive separation ratio $\psi$ and the importance of the role of normalized porosity were investigated theoretically and numerically in this paper. For stationary bifurcation, an analytical dispersion relation giving the critical Rayleigh number and wave number was obtained. Very good agreement was found between the critical values obtained analytically and the ones obtained by the spectral Tau numerical procedure. For Hopf bifurcations, the critical parameters depend strongly on the normalized porosity. The role of porosity is important. When it decreases, the stability of the equilibrium solution is reinforced.

For a cell heated from below, the equilibrium solution loses its stability via a stationary bifurcation for $\psi \in\left[\psi_{\mathrm{H}},+\infty\right]$ and for $\psi \leqslant \psi_{\mathrm{H}}=-1 /(L e(1+2 d \delta)+1)$ via Hopf bifurcation for the entire range of porosity values considered. For $\psi \in\left[\Psi_{\text {uni }},+\infty\right]$ the critical parameters associated with the primary transition are $R a_{\mathrm{cs}}=12(1+2 d \delta) /[1+\psi(2 d \delta L e+L e+1)]$ and $k_{\mathrm{cs}}=0$.

For a cell heated from above, the equilibrium solution is linearly stable if $\psi>0$, while the first bifurcation is stationary if $\psi<0$. 


\section{References}

Alonso A, Batiste O, Meseguer A and Mercader I 2007 Complex dynamical states in binary mixture convection with weak negative Soret coupling Phys. Rev. E 75026310

Batiste O, Knobloch E, Alonso A and Mercader I 2006 Spatially localized binary-fluid convection J. Fluid. Mech. 560 149-58

Bahloul A, Boutana N and Vasseur P 2003 Double-diffusive and Soret-induced convection in a shallow horizontal porous layer J. Fluid. Mech. 491 325-52

Bourich M, Hasnaoui M, Mamou M and Amahmid A 2004 Soret effect inducing subcritical and Hopf bifurcation in shallow enclosure filled with a clear binary fluid or saturated porous medium: a comparative study Phys. Fluids 16 551-68

Charrier-Mojtabi M C, Elhajjar B and Mojtabi A 2007 Analytical and numerical stability analysis of Soret-driven convection in a horizontal porous layer Phys. Fluids 19124104

Hurle D T J, Jakeman E and Pike E R 1967 On the solution of the Bénard problem with boundaries of finite conductivity Proc. R. Soc. A 296 469-75

Ingham D B and Pop I 2005 Transport Phenomena in Porous Media vol III (Amsterdam: North-Holland)

Jenkins D R and Proctor M R E 1984 The transition from roll to square-cell solutions in Rayleigh-Bénard convection J. Fluid Mech. 139 461-71

Jung D and Lücke M 2007 Bistability of moving and self-pinned fronts of supercritical localized convection structures Eur. Phys. Lett. 8014002

Knobloch E and Moore D R 1988 Linear stability of experimental Soret convection Phys. Rev. A 37 860-70

Mojtabi A and Rees D A S 2011 The effect of conducting bounding plates on the onset of Horton-Rogers-Lapwood convection Int. J. Heat Mass Transfer 54 293-301

Nield D A and Bejan A 2006 Convection in Porous Media 3rd edn (New York: Springer)

Ouarzazi M N and Bois P A 1994 Convection instability of a fluid mixture in a porous medium with time-dependent temperature-gradient Eur. J. Mech. B Fluids 13 275-98

Platten J K, Bou-Ali M M and Dutrieux J F 2003 Enhanced molecular separation in inclined thermogravitational columns J. Phys. Chem. B 107 11763-7

Proctor M R E 1981 Planform selection by finite-amplitude thermal convection between poorly conducting slabs J. Fluid Mech. 113 469-85

Rees D A S and Mojtabi A 2011 The effect of conducting boundaries on weakly nonlinear Darcy-Bénard convection Trans. Porous Med. 88 45-63

Riahi D N 1983 Nonlinear convection in a porous layer with finite conducting boundaries J. Fluid. Mech. 129 153-71

Schöpf W 1992 Convection onset for binary mixture in a porous medium and in a narrow cell: a comparison J. Fluid Mech. 245 263-78

Shevtsova V M, Melnikov D E and Legros J C 2006 Onset of convection in Soret driven instability Phys. Rev. E 73047302

Sovran O, Charrier-Mojtabi M C and Mojtabi A 2001 Naissance de la convection thermo-solutale en couche poreuse infinie avec effet Soret C. R. Acad. Sci. Paris, Ser. IIb, Mec des Fluides/Fluid Mechanics 329 287-93

Sparrow E M, Goldstein R J and Jonsson V H 1964 Thermal instability in a horizontal fluid layer: effect of boundary conditions and nonlinear temperature profile J. Fluid Mech. 18 513-28

Vafaï K 2005 Handbook of Porous Media 2nd edn (New York: Taylor and Francis) pp 269-320 